\title{
Comparação das Propriedades de Sorção e Solubilidade de Cimentos Submetidos a Diferentes Soluções e Tempos de Armazenagem
}

\author{
Priscilla do M. R. Busato, Erika C. S. de Oliveira, Mauro C. A. Busato, Márcio J. Mendonça, \\ Natiéli A. Rodrigues, Taline K. Vendrame, Veridiana Camilotti \\ Departamento de Odontologia, UNIOESTE
}

Resumo: A finalidade deste estudo foi avaliar in vitro algumas propriedades dos cimentos Rely X U100 (3M ESPE), Rely X Luting (3M ESPE) e All Cem (FGM), como a sorção e solubilidade em água destilada e numa solução de álcool $75 \%$, por períodos de 7 e 15 dias. Foram confeccionados 60 corpos-de-prova, divididos em três grupos $(\mathrm{n}=20)$, de acordo com o cimento escolhido. Eles foram então divididos em quatro grupos de acordo com os fatores avaliados, tipo de solução e período de avaliação. Os espécimes foram padronizados com $8 \mathrm{~mm}$ de diâmetro e $1 \mathrm{~mm}$ de espessura, sendo polimerizados sobre lâminas de microscopia e matriz de poliéster e os excessos foram removidos com lâmina de bisturi. As amostras foram transferidas para uma estufa a $37{ }^{\circ} \mathrm{C}$ até atingir a massa constante, denominada M1. Na sequência, foram imersas em suas respectivas soluções e permaneceram armazenadas por 7 e 15 dias. Decorrido este período, os espécimes foram pesados novamente, obtendo-se a M2. Para obter a nova massa constante, denominada M3, os mesmos retornaram à estufa a $37^{\circ} \mathrm{C}$. Para o cálculo da sorção e da solubilidade, foram utilizadas equações, sendo (M2-M3)/V e (M1-M3)/V, respectivamente. Os resultados foram submetidos à ANOVA de dois fatores.

Palavras-chave: Cimento resinoso, cimento ionomérico modificado por resinas, sorção, solubilidade.

\section{Comparison of Sorption and Solubility Properties of Cements Submitted to Different Solutions and Storage Times}

\begin{abstract}
The aim of this study was to evaluate in vitro some properties of the resin cement Rely X U100 (3M ESPE), such as the sorption and solubility in water and a solution of alcohol $75 \%$ for periods of 7 and 15 days. The 60 samples were divided into three groups according to the cement used, and then divided into four groups according to the factors evaluated, type of solution and evaluation period. The samples were standardized with $8 \mathrm{~mm}$ diameter and $1 \mathrm{~mm}$ in thickness and light cured in polyester matrix, with the excess being removed with a scalpel blade. The samples were transferred to a greenhouse at $37^{\circ} \mathrm{C}$ until reaching constant mass, referred to as M1. The samples were then immersed in solutions and stored for 7 and 15 days, after which they were weighed to get the mass M2. To reach a new constant mass, referred to as $\mathrm{M} 3$, the samples were again treated at $37^{\circ} \mathrm{C}$. The sorption and solubility were calculated using the equations (M2-M3)/V and (M1-M3)/V, respectively. The results were subjected to a two-way ANOVA procedure.
\end{abstract}

Keywords: Luting cements, resin modified ionomer cements, sorption, solubility.

\section{Introdução}

A odontologia moderna vem seguindo uma tendência de reduzir os passos operatórios no dia-a-dia do clínico, visando reduzir as dificuldades e simplificar a técnica. Como exemplo, temos o surgimento dos adesivos dentinários de frasco único e também os autocondicionantes. Seguindo essa mesma tendência surgiram no mercado, em 2002, uma nova geração de cimentos resinosos auto-adesivos, por apresentarem união química aos tecidos dentais, superfícies cerâmicas e metálicas e autocondicionantes, por dispensarem pré-tratamento no substrato e na restauração indireta ou prótese ${ }^{[1]}$. Esses cimentos estão disponíveis em sistema de ativação dupla, denominados dual, no qual existe associação dos processos de ativação química e de fotoativação, o que é considerado uma vantagem pois oferece melhoria nas propriedades físicas e mecânicas.

Estes cimentos foram constituídos inicialmente por um pó composto por partículas inorgânicas sólidas formadas de uma rede de vidro Al-Si-Na com incorporação de estrôncio e lantânio, e um líquido com um composto orgânico de ésteres metacrilatos fosfóricos associados à dimetacrilatos, acetatos, estabilizadores e iniciadores. Além disso, apresentam também em sua composição,
$2 \%$ de hidróxido de cálcio, o que pode induzir à mineralização, aumentar o efeito antimicrobiano e reduzir os níveis de acidez pós-presa, com diminuição da sensibilidade pós-operatória. Apresentam ainda $10 \%$ de fluoretos que podem contribuir para a redução de cáries recorrentes. O mecanismo de união ocorre pela quelação de íons cálcio por grupos ácidos, produzindo adesão química com a hidroxiapatita da estrutura dental. Quando estes sistemas são aplicado sobre a dentina, demostraram interação muito superficial sem a presença de camada híbrida ou tag's $\operatorname{resinosos}^{[2]}$.

Estes materiais são muito utilizados para procedimentos restauradores protéticos e sua efetividade relaciona-se à sua correta indicação clínica, combinado à suas propriedades físicas, mecânicas e biológicas ${ }^{[3]}$. Sua função é promover retenção entre a superfície interna da restauração e as irregularidades da estrutura dental, protegendo as margens de possível microinfiltração $\mathrm{O}^{[4]}$.

O cimento resinoso Rely X U100 é um cimento autocondicionante, que apresenta cargas silanizadas, metacrilatos e iniciadores. Embora a reação prevalente seja a polimerização iniciada pela luz, ocorre também uma reação ácido-base adicional 
que envolve adesão iônica e neutralização ácida pelo hidróxido de cálcio. Houve uma mudança na apresentação do produto para o sistema pasta-pasta, e o cimento antes denominado Unicem passou a ser chamado de U100, preservando porém a composição do cimento original ${ }^{[5]}$.

O Rely X Luting é um cimento ionomérico modificado por resina, composto por diferentes monômeros, sendo os principais HEMA e Bis-GMA. A polimerização do material acontece através de reação química e não de luz. Apresenta-se comercialmente como um sistema pasta-pasta embalados em um único frasco, denominado clicker, onde as quantidades saem iguais, minimizando assim, o erro por parte do operador durante a manipulação do material.

$\mathrm{O}$ cimento All Cem é um cimento resinoso adesivo de cura dual, ou seja, é quimicamente e fotoativado, portanto, permite a polimerização do material na presença ou ausência de luz. $O$ produto é composto por duas pastas: base e catalisadora. A pasta base confere pigmentação ao produto, enquanto a pasta catalisadora promove a polimerização química do cimento resinoso.

Os delineamentos experimentais sobre adesividade de alguns agentes cimentantes e as estruturas dentais relatam não haver a formação da camada híbrida e nem presença de tags resinosos com os substratos dentais, sendo que o aumento da resistência adesiva está na capacidade e efetividade do condicionamento e interação superficial com o esmalte e com a dentina ${ }^{[6,2]}$.

O desempenho clínico e a durabilidade de uma cimentação depende de muitos fatores, dependentes da ação da sorção e solubilidade como a estabilidade dimensional e a integridade estrutural. Falhas devido à deterioração das propriedades mecânicas e desgaste podem ser explicadas pela influência da umidade do meio bucal sobre o cimento resinoso, levando à degradação e subsequente microinfiltração ${ }^{[7]}$. Por esta razão, a sorção de água e o comportamento da solubilidade têm sido amplamente avaliados em ensaios clínicos e laboratoriais, porém devido à grande importância do tema, torna-se necessário que mais estudos sejam realizados ${ }^{[8,9]}$.

Vários fatores podem estar relacionados com a determinação da extensão em que um material se degrada no meio aquoso, dentre eles as características químicas, especialmente a hidrofilia do polímero e as diferenças entre o parâmetro de solubilidade do polímero e o solvente. Referente aos parâmetros estruturais, a densidade das ligações cruzadas, a porosidade da rede polimérica e a quantidade da carga do polímero exercem influência sobre a sorção e solubilidade da estrutura polimérica ${ }^{[14]}$.

$\mathrm{O}$ aspecto da solubilidade do material é também muito relevante na sua indicação, uma vez que clinicamente, pode levar à sua dissolução. Além dos efeitos deletérios às partículas inorgânicas, há a liberação de monômeros não reagidos ou subprodutos da degradação hidrolítica da matriz de resina, que além de ter impacto na biocompatibilidade do material, criam zonas que aumentam a retenção de água. Dentre os monômeros, o TEGDMA é o monômero mais solúvel das resinas compostas, já o Bis-GMA e o UDMA podem ser liberados caso o grau de conversão do material resinoso esteja comprometido ${ }^{[7]}$.

Desta forma, o objetivo do presente estudo foi testar a sorção e solubilidade dos cimentos Rely X U100 (3M ESPE), Rely X Luting (3M ESPE) e All Cem (FGM), após armazenamento em duas soluções: água e água mais álcool (25-75\%), por diferentes períodos, 7 e 15 dias.

\section{Materiais e Métodos}

Foram confeccionados sessenta corpos-de-prova, seguindo as instruções do fabricante, sendo divididos em três grupos $(n=20)$, de acordo com o agente cimentante, G1- Rely X U100, G2 - Rely X Luting e G3 - All Cem. Os materiais foram inseridos com o auxílio

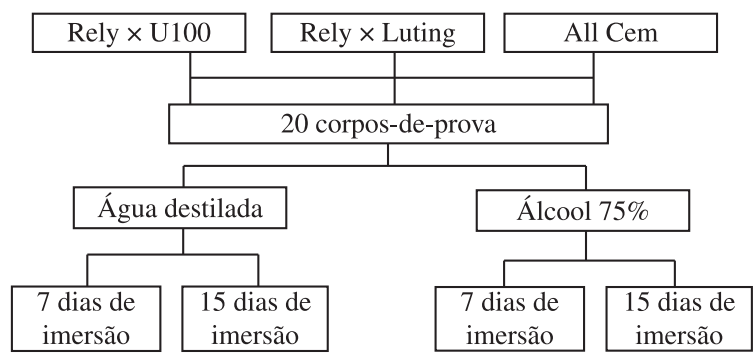

Figura 1. Divisão dos corpos-de-prova.

de uma seringa Centrix (Centrix ${ }^{\mathrm{TM}}$ ), em uma matriz de silicona de adição (Adsil, Vigodent), com $8 \mathrm{~mm}$ de diâmetro por $1 \mathrm{~mm}$ de espessura e o excesso foi removido posicionando-se uma tira de poliéster e em seguida uma placa de vidro, possibilitando dessa forma, a obtenção de uma superfície plana, lisa e totalmente polimerizada. A fotoativação foi realizada com um aparelho fotopolimerizador de lâmpada LED cuja irradiância é de $900 \mathrm{~mW} \cdot \mathrm{cm}^{-2}$ (Free Light II - 3M ESPE). Os corpos-de-prova foram removidos imediatamente após a polimerização e possíveis excessos laterais foram removidos com lâmina de bisturi número 12. As amostras foram então armazenadas em uma estufa (Lab-line Imperial II) a $37^{\circ} \mathrm{C}$ contendo sílica gel e os mesmos foram pesados a cada 24 horas em uma balança analítica (Mettler, Toledo) de precisão de 0,01 $\mathrm{mg}$, até que se obteve uma massa constante, denominada de M1. Após a obtenção da M1, as amostras foram imersas separadamente em recipientes de $5 \mathrm{ml}$ cada contendo água destilada ou solução de álcool (75\%) mais água destilada (25\%) permanecendo armazenadas por 7 e 15 dias (Figura 1) e depois armazenadas em estufa a $37{ }^{\circ} \mathrm{C}$. Decorrido este período, as amostras foram retiradas das soluções e secas com papel absorvente e pesadas na balança analítica, obtendo-se a M2. Depois disso, as amostras voltaram ao dissecador por 7 e 15 dias à $37^{\circ} \mathrm{C} \mathrm{e}$ após o terceiro ciclo de pesagem, também realizado a cada 24 horas, foi obtida a M3. Após esta etapa, a espessura das amostras foi medida em três pontos através de um paquímetro digital (Starret ${ }^{\mathrm{TM}}$ ) para o cálculo do volume, aplicando-se a fórmula $\mathrm{V}=\varpi \mathrm{r}^{2} \mathrm{xe}$, (onde $r=$ raio e e = espessura). Os valores para sorção e solubilidade em água foram calculados em $\mu \mathrm{g} \cdot \mathrm{mm}^{-3}$, através de equações numéricas (M2-M3/V) e (M1-M3/V), respectivamente.

Para a análise estatística dos resultados, utilizou-se o teste de variância de dois fatores.

\section{Resultados e Discussão}

O tempo de armazenagem utilizado neste trabalho, de 7 e 15 dias, é considerado um período suficiente para a observação do grau de sorção, já que segundo Chutinan et al. (2004), é nas duas primeiras semanas que acontece mais intensamente a sorção de água ${ }^{[11]}$. Além disso, Gerdolle et al. (2008), observaram que os cimentos resinosos apresentam pouca alteração pós polimerização, o que explica a mínima alteração na absorção de água entre 4 e 7 dias, justificando que o tempo de 7 e 15 dias são suficientes e ideais para a análise ${ }^{[12]}$.

Para que os cimentos resinosos fossem simplificados e conseguissem suprir o passo do condicionamento ácido do substrato no processo adesivo, sua composição sofreu modificações que podem tê-lo deixado mais suscetíveis à umidade, ou seja à sorção, constituindo um problema clínico ${ }^{[13]}$. Uma característica desejável dos cimentos resinosos e ionoméricos é a estabilidade após a polimerização e não interação com o meio quando exposto à cavidade bucal. Entretanto, a maioria dos monômeros resinosos empregados nesses materiais apresentam caráter hidrofílico e frequentemente sofrem sorção de água. 
Tabela 1. Composição química dos cimentos.

\begin{tabular}{|c|c|c|c|}
\hline Cimento & Característica & Sistema de distribuição & Composição química \\
\hline Rely X U100 & $\begin{array}{l}\text { Dual } \\
\text { Auto-adesivo } \\
\text { Autocondicionante }\end{array}$ & $\begin{array}{l}\text { Clicker dispenser - } 2 \text { pastas } \\
\text { (1 pasta base e } 1 \text { pasta } \\
\text { catalisadora) }\end{array}$ & $\begin{array}{l}\text { Base: fibra de vidro, ácido fosfórico metacrilato, dimetacrilato, } \\
\text { sílica silanizada, persulfato de sódio. } \\
\text { Catalisador: fibra de vidro, dimetacrilato, sílica silanizada, } \\
\text { P-tolueno, sulfato de sódio, hidróxido de cálcio. } \\
\text { TEGDMA } 10-20 \% \\
72 \% \text { carga inorgânica } \\
90 \% \text { partículas }<9,5 \mu \mathrm{m}\end{array}$ \\
\hline Rely X Luting & $\begin{array}{l}\text { Cimento de ionômero de vidro } \\
\text { Modificado por resina }\end{array}$ & $\begin{array}{c}\text { Clicker dispenser - } 2 \text { pastas } \\
\text { (1 pasta base e } 1 \text { pasta } \\
\text { catalisadora) }\end{array}$ & $\begin{array}{l}\text { Pasta A: Vidro fluor-alumínio-silicato, agente redutor, agente } \\
\text { opacificante HEMA, água. Pasta B:ácido policarboxilico } \\
\text { metacrilatado, Bis-GMA, HEMA, água, perssulfato de potássio, } \\
\text { carga de zircônia, sílica. }\end{array}$ \\
\hline All Cem & $\begin{array}{c}\text { Cimento resinoso } \\
\text { Dual }\end{array}$ & $\begin{array}{l}\text { Seringa de corpo duplo: pasta } \\
\text { base + pasta catalisadora }\end{array}$ & $\begin{array}{l}\text { Bis-GMA, Bis-EMA, TEGDMA, canforquinona, peróxido de } \\
\text { dibenzoila e estabilizantes. } \\
\text { Micropartículas de vidro de bário-alumino silicato e nanopartículas } \\
\text { de dióxido de silício. }\end{array}$ \\
\hline
\end{tabular}

De acordo com os resultados obtidos neste trabalho, pôde-se observar que não houve diferença para a quantidade de sorção dos três cimentos avaliados quando se consideram os períodos de 7 e 15 dias (Tabela 2).

No período de 7 dias de armazenagem, o cimento All Cem, tanto em meio aquoso quanto em meio alcoólico, apresentou menores índices de sorção do que os outros dois cimentos testados, embora do ponto de vista estatístico, esta diferença tenha sido realmente significante apenas quando o All Cem foi comparado ao Rely X Luting em meio aquoso.

No período de 15 dias, o cimento Rely X Luting apresentou um alto índice de sorção, principalmente em meio aquoso, fato este que pode ser mais observado ainda quando comparado ao cimento All Cem, que demonstrou baixos índices de sorção tanto em meio aquoso quanto em meio alcoólico.

$\mathrm{O}$ cimento Rely X Luting, que é um material ionomérico modificado por resina, exibiu, de uma forma geral, uma maior sorção quando comparado ao Rely X U100 e ao All Cem, que são classificados como cimentos resinosos polimerizáveis, tanto em água como em álcool, achados estes, similares aos encontrados por Mese et al. (2008). Além disso, Knobloch et al. (2000) encontraram resultados semelhantes ao deste trabalho, onde observaram que os cimentos de ionômero de vidro modificados por resina exibiram entre 8 e $14 \%$ da absorção de água por peso ${ }^{[14]}$.

A implicação clínica deste achado é que a sorção de água aumentada encontrada nos cimentos à base de ionômero de vidro pode resultar em uma expansão higroscópica, cujos efeitos iniciais podem ser benéficos ao compensarem qualquer contração de polimerização, mas ao longo do tempo podem levar a efeitos adversos, que atuam tanto sobre a estrutura dentária quanto sobre a restauração. O principal monômero resinoso presente no cimento de ionômero de vidro é o hidroxietil metacrilato (HEMA). Acredita-se que a sua presença na composição do material seja o principal motivo para a sorção e consequente expansão higroscópica ${ }^{[15]}$. Devido a este fator, o uso do cimento de ionômero de vidro modificado por resina para cimentar coroas de cerâmica pura, torna-se contra-indicado, já que diversas fraturas da peça protética têm sido associadas à ele ${ }^{[16]}$.

Quando as amostras de cimento são imersas em água, alguns dos componentes, como os monômeros, dissolvem-se e migram para a água, resultando em perda de peso e volume que pode ser medido como solubilidade ${ }^{[17,18,19]}$. A eluição desses componentes pode influenciar a alteração dimensional inicial dos cimentos à base de resinas, comprometendo a estética e a biocompatibilidade ${ }^{[7,10]}$.
Tabela 2. Valores médios de sorção e respectivos desvios-padrão para os cimentos resinosos em função das soluções, nos diferentes períodos de leitura $\left(\mu \mathrm{g} \cdot \mathrm{mm}^{-3}\right)$.

\begin{tabular}{cccc}
\hline Soluções & \multicolumn{2}{c}{ Período } & Material \\
\cline { 2 - 3 } & $\mathbf{7 ~ d i a s}$ & $\mathbf{1 5}$ dias & \\
\hline Água & $86,3( \pm 47,4) \mathrm{Aa}$ & $93,4( \pm 70,1) \mathrm{Aab}$ & \multirow{2}{*}{ Rely X Luting } \\
Álcool & $23,7( \pm 13,3) \mathrm{Aab}$ & $156,0( \pm 014,0) \mathrm{Aa}$ & \\
\hline Água & $32,7( \pm 5,09) \mathrm{Aab}$ & $43,2( \pm 4,35) \mathrm{Aab}$ & \multirow{2}{*}{ Rely X U 100 } \\
Álcool & $36,8( \pm 5,08) \mathrm{Aab}$ & $45,9( \pm 2,86) \mathrm{Aab}$ & \\
\hline Água & $-6,46( \pm 8,86) \mathrm{Ab}$ & $12,0( \pm 1,50) \mathrm{Ab}$ & \multirow{2}{*}{ All Cem } \\
Álcool & $6,01( \pm 1,46) \mathrm{Ab}$ & $12,8( \pm 0,719) \mathrm{Ab}$ & \\
\hline
\end{tabular}

Médias seguidas por letras distintas diferem entre si (maiúsculas na horizontal e minúsculas na vertical - $\mathrm{p} \leq 0,05$ ).

Tabela 3. Valores médios de solubilidade e respectivos desvios-padrão para os cimentos resinosos em função das soluções, nos diferentes períodos de leitura $\left(\mu \mathrm{g} \cdot \mathrm{mm}^{-3}\right)$.

\begin{tabular}{cccc}
\hline Soluções & \multicolumn{2}{c}{ Período } & Material \\
\cline { 2 - 3 } & $\mathbf{7 ~ d i a s}$ & $\mathbf{1 5}$ dias & \\
\hline Água & $32,9( \pm 4,15) \mathrm{Aa}$ & $5,69( \pm 15,3) \mathrm{Aab}$ & \multirow{2}{*}{ Rely X Luting } \\
Álcool & $8,74( \pm 2,42) \mathrm{Aa}$ & $7,10( \pm 18,8) \mathrm{Aab}$ & \\
\hline Água & $2,70( \pm 0,700) \mathrm{Aa}$ & $4,50( \pm 1,00) \mathrm{Aab}$ & \multirow{2}{*}{ Rely X U 100 } \\
Álcool & $20,9( \pm 6,12) \mathrm{Aa}$ & $27,7( \pm 4,94) \mathrm{Aa}$ & \\
\hline Água & $-6,46( \pm 8,86) \mathrm{Aa}$ & $-4,80( \pm 0,552) \mathrm{Ab}$ & \multirow{2}{*}{ All Cem } \\
Álcool & $0,30( \pm 1,65) \mathrm{Aa}$ & $-3,92( \pm 0,915) \mathrm{Aab}$ & \\
\hline
\end{tabular}

Médias seguidas por letras distintas diferem entre si (maiúsculas na horizontal e minúsculas na vertical - $\mathrm{p} \leq 0,05)$.

Com relação à solubilidade, também não houve diferença quando se considera os períodos de 7 e 15 dias, para nenhum dos cimentos testados (Tabela 3 ).

Não houve diferença para a quantidade de solubilidade quando se consideram os cimentos avaliados, no período de sete dias. Já no período de 15 dias, o Rely X U 100 apresentou em meio alcoólico, maiores índices de solubilidade do que os outros dois cimentos testados, embora do ponto de vista estatístico, esta diferença tenha sido realmente marcante quando o Rely X U100 foi comparado ao All Cem, sendo este em meio aquoso e meio alcoólico.

O All Cem também exibiu uma menor solubilidade quando comparado aos outros dois cimentos estudados, principalmente quando foram considerados os períodos de 15 dias e os dois meios de armazenagem. 
Como os grupos de radicais livres polimerizáveis dos cimentos resinosos são atacados pelas cadeias de ácido policarboxílico, há uma diminuição na probabilidade de monômeros livres serem transportados para fora do meio aquoso ${ }^{[15]}$, explicando-se assim, os resultados de menor solubilidade encontrados para o cimento All Cem. Subsequentemente, a natureza hidrofílica dos cimentos ionoméricos contribui de maneira importante para uma maior solubilidade deste tipo de cimento quando comparado aos cimentos $\operatorname{resinosos}^{[15]}$.

Dentre os monômeros, o TEGDMA é o monômero mais solúvel das resinas compostas, já o Bis-GMA e o UDMA podem ser liberados caso o grau de conversão do material resinoso esteja comprometido $^{[21]}$, o que justifica os altos resultados de solubilidade encontrados para o cimento Rely X U 100.

Foram encontrados valores negativos para a solubilidade do cimento All Cem, o que está de acordo com o encontrado na literatura. Segundo Vrochari, os resultados negativos demonstram que não houve retirada de componentes pelo processo de dissolução, afirmando que a quantidade de água que permeneceu vinculada à matriz foi superior à quantidade de componentes retirados pelo processo de dissolução, o que sugere um ganho de massa aparente. Este fato também está de acordo com o trabalho de Lopes et al. (2008), que também encontraram valores de solubilidade negativos, o que indica que o cimento é mais sensível à sorção de água levando ao ganho de massa, que poderia mascarar a solubilidade real. Isso não significa que não ocorreu solubilidade, mas que a sorção de água foi maior que a solubilidade ${ }^{[22]}$.

Tanto a sorção quanto a solubilidade são dependentes da composição química dos materiais resinosos. Os monômeros BisGMA, TEGDMA e UDMA são os que mais influenciam essas propriedades. Segundo Ortengren et al. (2001), materiais que possuíam BisGMA na sua composição foram os que mais sofreram sorção, enquanto que os cimentos com TEGDMA foram os que mais sofreram solubilidade ${ }^{[23]}$. Esses resultados concordam com os que foram encontrados no presente estudo, já que o TEGDMA é um componente do cimento Rely X U100 e este material demonstrou maior solubilidade quando se comparou os períodos de avaliação e também quando se levou em consideração as soluções testadas, apesar de nesse último caso, não ter havido diferença estatisticamente significante. Além disso, o cimento Rely X Luting, que contém BisGMA na sua composição, foi o que apresentou os maiores valores de sorção, independente das soluções ou dos períodos avaliados.

\section{Conclusão}

Com base nos resultados obtidos neste estudo, pôde-se concluir que o cimento ionomérico Rely $\mathrm{X}$ Luting apresentou maiores valores de sorção independente do período de armazenagem.

$\mathrm{O}$ cimento All Cem foi o que apresentou menor grau de solubilidade, tanto para o período quanto para a solução testada.

Com relação ao tempo de armazenagem, observou-se que não houve diferença nos valores de sorção e solubilidade entre 7 e 15 dias para nenhum dos cimentos testados.

\section{Referências Bibliográficas}

1. Reich, S. M.; Wichmann, M.; Frankenberger, R. \& Zaj, D. - J. Biomed. Mater. Res., B Appl. Biomater., 74, p. 704 (2005).

2. Al Assaf, K.; Chakmakchi, M.; Palaghias, G.; Karanica-Kouma, A. \& Eliades, G. - Dent. Mater., 23, p. 829 (2007). PMid:16934865. http:// dx.doi.org/10.1016/j.dental.2006.06.023
3. Nakabayashi, N.; Kojima, K. \& Masuhara, E. - J. Biomed. Mater. Res., 16, p. 265 (1982) . PMid:7085687. http://dx.doi.org/10.1002/ jbm.820160307

4. Burke, F. J. - Dent. Update., 32, p. 251 (2005). PMid:15977720.

5. 3M ESPE. - "Rely X U100 self adhesive universal resin cement", 3M ESPE, Germany (2008).

6. De Munck, J.; Van Meerbeek, B.; Yoshida, Y.; Inoue, S.; Vargas, M.; Suzuki, K.; Lambrechts, P. \& Vanherle, G. - J. Dent. Res., 82, p. 136 (2003). PMid:12562888. http://dx.doi. org/10.1177/154405910308200212

7. Ortengren, U.; Elgh, U.; Spasenoska, V.; Karlsson, S.; Milleding, P. \& Hassum, J. - Int. J. Prosthodont., 13, p. 141 (2000) . PMid:11203623.

8. Kanchanavasita, W.; Anstice, H. M. \& Pearson, G. J. - Biomaterials, 18, p. 343 (1997). http://dx.doi.org/10.1016/S0142-9612(96)00124-X

9. Knobloch, L. A.; Kerby, R. E.; McMillen, K. \& Clelland, N. - Oper. Dent., 25, p. 434 (2000).

10. Ferracane, J. L. - J. Oral. Rehabil., 21, p. 441 (1994) . PMid:7965355. http://dx.doi.org/10.1111/j.1365-2842.1994.tb01158.x

11. Chutinan, S.; Platt, J. A.; Cochran, M. A.; Moore, B. K. - Dent. Mater., 20, p. 345 (2004). http://dx.doi.org/10.1016/S01095641(03)00127-1

12. Gerdolle, D. A.; Mortier, E.; Jacquot, B. \& Panighi, M. M. Quintessence Int., 39, p. 265 (2008).

13. Chersoni, S.; Suppa, P.; Grandini, S.; Goracci, C.; Monticelli, F.; Yiu, C.; Huang, C.; Prati, C.; Breschi, L.; Ferrari, M.; Pashley, D. H. \& Tay, F. R. - J. Dent. Res., 83, p. 459 (2004). PMid:15153452. http://dx.doi. org/10.1177/154405910408300605

14. Knobloch, L. A.; Kerby, R. E.; McMillen, K. \& Clelland, N. - Oper. Dent., 25, p. 434 (2000). PMid:11203853.

15. Yap, A. \& Lee, C. M. - J. Oral Rehabil., 24, p. 310 (1997). PMid:9147305. http://dx.doi.org/10.1046/j.1365-2842.1997.d01-282.x

16. Leevailoj, C.; Platt, J. A.; Cochran, M. A. \& Moore, B. K. - J. Prosthet. Dent., 80, p. 699 (1998). http://dx.doi.org/10.1016/S00223913(98)70058-7

17. Toledano, M.; Osório, R.; Osório, E.; Fuentes, V.; Prati, C. \& GarciaGodoy, F. - J. Dent., 31, p. 43 (2003). http://dx.doi.org/10.1016/S03005712(02)00083-0

18. Mohsen, N. M. \& Craig, R. G. - J. Oral Rehabil., 22, p. 213 (1995). PMid:7769516. http://dx.doi.org/10.1111/j.1365-2842.1995.tb01566.x

19. Burrow, M. F.; Inokoshi, S. \& Tagami, J. - Am. J. Dent., 12, p. 295 (1999). PMid:10850250.

20. Iwami, Y.; Yamamoto, H.; Sato, W.; Kawai, K.; Torii, M. \& Ebisu, S. Oper. Dent., 23, p. 132 (1998). PMid:9656924.

21. Vrochari, A. D., Eliades, G., Hellwig, E.\& Wrbas, K. - J. Ades. Dent., 12, p.39 (2010).

22. Lopes, L. G., Jardim Filho, A. V., Souza, J. B., Rabelo, D., Franco, E. B.\& Fraitas, G. C. - J. Appl. Oral Sci., 17, p.27 (2009). http://dx.doi. org/10.1590/S1678-77572009000100006

23. Ortengren, U., Andersson, F., Elgh, U., Terselius, B. \& Karlsson, S. - J. Dent., 29, p.35 (2001). http://dx.doi.org/10.1016/S03005712(00)00055-5

Enviado: $17 / 11 / 10$

Reenviado: 15/03/11

Aceito: 11/05/11 